\title{
Prediction model of sinoatrial node field potential using high order partial least squares
}

\author{
Yu Feng, Hui Cao* and Yanbin Zhang \\ State Key Laboratory of Electrical Insulation and Power Equipment, School of Electrical Engineering, \\ $X i$ 'an Jiaotong University, Xi'an, 710049, China
}

\begin{abstract}
High order partial least squares (HOPLS) is a novel data processing method. It is highly suitable for building prediction model which has tensor input and output. The objective of this study is to build a prediction model of the relationship between sinoatrial node field potential and high glucose using HOPLS. The three sub-signals of the sinoatrial node field potential made up the model's input. The concentration and the actuation duration of high glucose made up the model's output. The results showed that on the premise of predicting two dimensional variables, HOPLS had the same predictive ability and a lower dispersion degree compared with partial least squares (PLS).
\end{abstract}

Keywords: High order partial least squares, sinoatrial node field potential, prediction model, signal decomposition

\section{Introduction}

In biomedical engineering research, the processing of electrophysiological data is a significant field. The amount of experimental data has increased sharply since the fast development and extensive application of multiple electrodes electrophysiological sensors [1-3]. When the amount of data is huge, some features may be hidden in the data and be difficult to find. In this case, researchers tend to pay more attention to find the internal relations of the data and then evaluate the new experimental data using these relations. Therefore, accurate prediction models need to be built.

The partial least square (PLS) is a classical and widely used modeling method which can predict a dependent variable set from an independent variable set. There are many variations of the PLS model such as recursive PLS, biorthogonal PLS and nonlinear PLS. These methods have been used widely to process electrophysiological signals [4-6]. Most of the methods, however, have strict requirements for the input and output of the models. For example, the input must be a matrix and the output must be a vector. In fact, most of the experimental data describe the relations between multi-way input and multi-way output which are deemed as tensors. In order to adjust this kind of data, a new prediction model, higher order partial least squares (HOPLS), was proposed [7, 8]. HOPLS is highly suitable for

\footnotetext{
* Address for correspondence: Hui Cao, Industrial Automation Department, Electrical Engineering School, Xi'an Jiaotong University, West Xianning Road, 28\#, Xi'an, Shaanxi, 710049, P.R. China. Tel.: +86 029 82668666-212; Fax: +86 029 83237910; E-mail: huicaoxjtu@sina.com.
} 
building a prediction model which has tensor-input and tensor-output. The model can predict several variables synchronously.

In this paper, HOPLS was used to build a prediction model which could predict the concentration and actuation duration of high glucose to the sinoatrial node field potential. The sinoatrial node is the pacemaker tissue located in the right atrium of the heart [9]. The sinoatrial node field potential is an important electrophysiological signal which could reflect the conduction law of cardiac pacing signals. The data preprocessing results showed that the sinoatrial node field potential consisted of three different sub-signals. The three signals had different sensitivities to high glucose and did not disturb each other. The HOPLS prediction model could predict the concentration and actuation duration of high glucose according to the sub-signals. Compared with the prediction results of PLS, the predictive ability of HOPLS had the same level of root mean squared error of prediction (RMSEP) and a lower dispersion degree.

The paper is organized as follows: Part 2 presents the modeling methods of PLS and HOPLS, as well as the biology experimental equipment and processes. Part 3 presents the results and discussions of the biology experiments and prediction models. Part 4 is the conclusion.

\section{Materials and methods}

\subsection{Prediction modeling methods}

In this paper, $N$ th-order tensors are denoted by underlined boldface capital letters, e.g., $\underline{\mathbf{X}}$; matrices by boldface capital letters, e.g., $\mathbf{Y}$; vectors by boldface lower case letters, e.g., $\mathbf{v}$ and scalars by italic lower case letters, e.g. $a$. The $i$ th entry of a vector $\mathbf{v}$ is denoted by $v_{i}$; element $(i, j)$ of a matrix $\mathbf{Y}$ by $y_{i j}$; and element $\left(i_{1}, i_{2}, \ldots, i_{N}\right)$ of an $N$ th-order tensor $\underline{\mathbf{X}}\left(\underline{\mathbf{X}} \in \mathrm{IR}^{I_{1} \times I_{2} \times \ldots \times I_{N}}\right)$ by $x_{i_{1} i_{2} \ldots i_{N}}$ or $(\underline{\mathbf{X}})_{i_{1} i_{2} \ldots i_{N}}$. The $n$-mode product of tensor $\underline{\mathbf{X}} \in \mathrm{IR}^{I_{1} \times I_{2} \times \ldots \times I_{n} \times \ldots \times I_{N}}$ and matrix $\mathbf{A} \in \mathrm{IR}^{I_{n} \times I_{n}}$ is denoted by $\underline{\mathbf{Y}}=\mathbf{X} \times{ }_{n} \mathbf{A} \in$ $\mathrm{IR}^{I_{1} \times I_{2} \times \ldots \times I_{n-1} \times I_{n} \times I_{n+1} \times \ldots \times I_{N}}$ and is defined as

$$
y_{i_{1} i_{2} \ldots i_{n-1} j_{n} i_{n+1} \ldots i_{N}}=\sum_{i_{n}} x_{i_{1} i_{2} \ldots i_{n} \ldots i_{N} a_{j_{n} i_{n}}}
$$

For PLS, we calculated the output variables $\mathbf{Y}$ by Eq. (2):

$$
\mathbf{Y}=\mathbf{X D}+\mathbf{r}
$$

where $\mathbf{X}$ is an input variable quantity, $\mathbf{D}$ is a regression coefficient matrix, and $\mathbf{r}$ is a bias vector.

When a small number of principal components are defined by linear combinations of the input matrix, Eq. (2) can be rewritten by Eq. (3):

$$
\mathbf{Y}=\mathbf{T} \mathbf{v}+\mathbf{r}
$$

where $\mathbf{v}$ is a vector of regression coefficients corresponding to the latent variables.

Then the matrix $\mathbf{T}$ can be calculated by Eq. (4):

$$
\mathbf{T}=\mathbf{X W}\left(\mathbf{P}^{\mathbf{T}} \mathbf{W}\right)^{-1}
$$


where $\mathbf{P}$ is the loading matrix representing the influence of $\mathbf{X} . \mathbf{W}$ is the weight loading matrix indicating the correlation between $\mathbf{Y}$ and $\mathbf{X}$.

For HOPLS, $\underline{\mathbf{X}}$ is decomposed as a sum of rank- $\left(1, L_{2}, \ldots, L_{N}\right)$ Tucker blocks. $\underline{\mathbf{Y}}$ is decomposed as a sum of rank-(1, $\left.\bar{K}_{2}, \ldots, K_{M}\right)$ Tucker blocks [7]. $\underline{\mathbf{X}}$ and $\underline{\mathbf{Y}}$ can be written by Eq. (5):

$$
\begin{aligned}
& \underline{\mathbf{X}}=\sum_{r=1}^{R} \underline{\mathbf{G}}_{r} \times_{1} \mathrm{t}_{r} \times_{2} \mathbf{P}_{r}^{(1)} \times_{3} \ldots \times_{N} \mathbf{P}_{r}^{(N-1)}+\underline{\mathbf{E}}_{r} \\
& \underline{\mathbf{Y}}=\sum_{r=1}^{R} \underline{\mathbf{D}}_{r} \times_{1} \mathbf{t}_{r} \times_{2} \mathbf{Q}_{r}^{(1)} \times_{3} \ldots \times_{M} \mathbf{Q}_{r}^{(M-1)}+\underline{\mathbf{F}}_{r}
\end{aligned}
$$

where $R$ is the latent vectors' number, $\mathbf{t}_{r} \in \mathrm{IR}^{I_{1}}$ is the $r$ th latent vector, $\left\{\mathbf{P}_{r}^{(n)}\right\}_{n=1}^{N-1} \in \mathrm{IR}^{I_{n+1} \times L_{n+1}}$ and $\left\{\mathbf{Q}_{r}^{(m)}\right\}_{n=1}^{M-1} \in \mathrm{IR}^{J_{m+1} \times K_{m+1}}$ are loading matrices on mode- $n$ and mode- $m, \underline{\mathbf{G}}_{r}$ and $\underline{\mathbf{D}}_{r}$ are core tensors. $\underline{\mathbf{G}}_{r} \in \mathrm{IR}^{1 \times L_{2} \times \ldots \times L_{N}}, \underline{\mathbf{D}}_{r} \in \mathrm{IR}^{1 \times K_{2} \times \ldots \times K_{M}}$.

For the three-way independent variables $\underline{\mathbf{X}} \in \mathrm{IR}^{I \times J \times K}$ and the dependent variable $\mathbf{Y} \in \mathrm{IR}^{I \times M}$ with the same sample size $I$, the model can be simplified as Eq. (6):

$$
\begin{gathered}
\underline{\mathbf{X}}=\sum_{r=1}^{R} \underline{\mathbf{G}}_{r} \times_{1} \mathbf{t}_{r} \times_{2} \mathbf{P}_{r} \times_{3} \mathbf{S}_{r}+\underline{\mathbf{E}}_{r} \\
\mathbf{Y}=\sum_{r=1}^{R} d_{r r} \mathbf{t}_{r} \mathbf{c}_{r}^{T}+\underline{\mathbf{F}}_{r}
\end{gathered}
$$

where $\mathbf{P}_{r} \in \mathrm{IR}^{J \times L_{2}}\left(J \geq L_{2}\right), \mathbf{S}_{r} \in \mathrm{IR}^{K \times L_{3}}\left(K \geq L_{3}\right)$ are the loading matrices corresponding to the latent vector $\mathbf{t}_{r}$.

If we define $\underline{\mathbf{Z}}=\underline{\mathbf{X}} \times{ }_{1} \mathbf{Y}$, the parameters $\mathbf{P}, \mathbf{S}, \mathbf{c}$ can be learned by maximizing the objective function

$$
\max _{\mathbf{P}, \mathbf{S}, \mathbf{c}}\left\|\underline{\mathbf{G}} \times{ }_{1} \mathbf{c}^{T} \times{ }_{2} \mathbf{P}^{T} \times{ }_{3} \mathbf{S}^{T}\right\|^{2}, \text { subject to } \mathbf{P}^{T} \mathbf{P}=\mathbf{I}_{L_{2}}, \mathbf{S}^{T} \mathbf{S}=\mathbf{I}_{L_{3}} \text { and }\|\mathbf{c}\|=1
$$

\subsection{Animals}

Male C57/BL6J mice which were fed a standard laboratory diet and water were chosen for the experiments. The mice were 8-12 weeks old and weighed 20-25 g. The ambient temperature was $21^{\circ} \mathrm{C} \pm 2{ }^{\circ} \mathrm{C}$. The Ethics Committee of Animal Experimentation approved the experimental protocol, and the study was performed according to the Guidelines for Animal Experimentation of Xi'an Jiaotong University.

\subsection{Recording instruments}

The MED64 Recording and Analysis System (Panasonic Alpha Med Science Company, Japan) were used to record and analyze the experimental data. The system comprised an MED64 amplifier, an MED64 connector, an MED64 controller, a data processing computer, an inverted microscope, a perfusion cap, a peristaltic pump and a measuring electrode. The electrode had 64 channels which 
were arranged in $8 \times 8$ squares. The electrode spacing was $300 \mu \mathrm{m}$. Each electrode was square and the length of each side was $50 \mu \mathrm{m}$.

\subsection{Design of the experiment}

To begin, mouse heart was needed in vitro perfusion. The Langendorff system [10] and Tyrode's solution [11] were used for the perfusion. Afterwards, the sinoatrial node located on the right atrium [12] was cut off at a thickness of $1 \mathrm{~mm}$. Then the sample was directly placed into the MEA electrode for recording. In the entire recording process, Tyrode's solution with 5\% carbon dioxide and 95\% oxygen was provided continuously with a flow rate of $5 \mathrm{ml} / \mathrm{min}$.

After recording the field potentials of the control sample, high glucose solutions were fed into the sample at 0 th minute. The concentrations were $20 \mathrm{mM}, 30 \mathrm{mM}, 40 \mathrm{mM}$ and $50 \mathrm{mM}$, respectively at a duration of 30 minutes. Data were recorded every five minutes beginning from the 10th minute. The sampling frequency was $20 \mathrm{kHz}$. Meanwhile, mannitol solutions with identical concentrations were fed into the iso-osmotic group to eliminate the possible effects of high osmotic pressure. The results showed that the field potential was not sensitive to high osmotic pressure.

Fast Fourier transform (FFT) was used to convert the field potential signals from the time domain to the frequency domain. Most of the power of the frequency domain signals was focused at $0-50 \mathrm{~Hz}$. Data from this section were selected as the experimental data. The dataset contained 1166 samples. Shutter grouping strategy [13] was used to split the dataset into the validation set and the calibration set. For every two samples, one was placed into the calibration set and the other was placed into the validation set. The calibration set was used to build the prediction model. The validation set was used to estimate the effectiveness of the modeling method.

\section{Results and discussion}

\subsection{Data preprocessing}

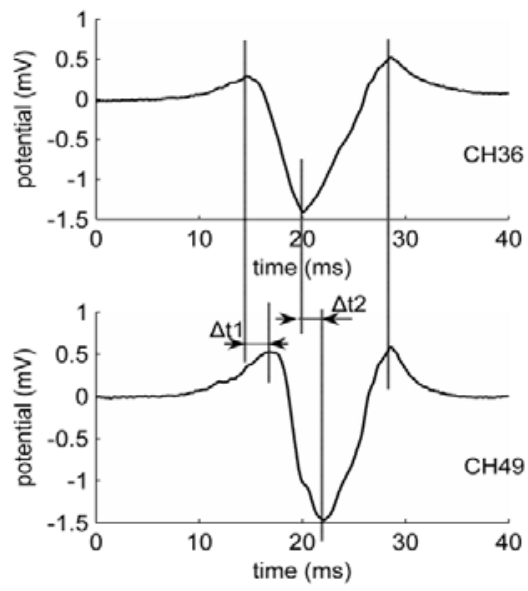

(a)

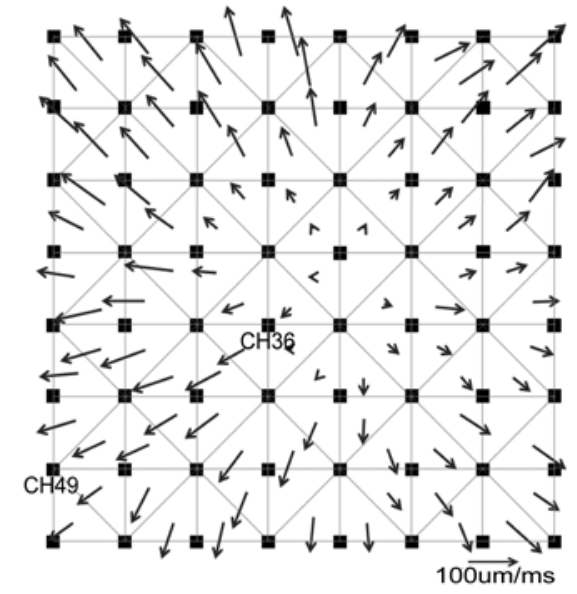

(b)

Fig. 1. Conductive characteristics of the field potentials in the time domain. (a) The signals gotten from two recording points (Channel 36 and Channel 49). The spacing was $1 \mathrm{~mm}$. The conducting direction was from Channel 36 to Channel 49. (b) The velocity vector map of the first positive wave peak. 
Figure 1(a) showed signals in the same cycle from two different recording points. Both of the first two wave peaks had time intervals ( $\Delta \mathrm{t} 1$ and $\Delta \mathrm{t} 2)$, which meant that these two kinds of wave peaks were conductive. Figure 1(b) showed the conducting directions and velocities [14] of the first positive wave peak. In the center, there were no time intervals. It could be considered that in this area, the characteristic of the signals occurred simultaneously. Apart from the centre, the farther the electrodes were from the area, the later the wave peaks occurred. It showed that the characteristics of the signals spread around from the center. The negative wave peak had a similar conduction mode. On the contrary, the second positive wave peaks appeared almost at the same time point in the entire recording area. It indicated that the sinoatrial node field potentials had two kinds of different conductive characteristics. The signal was composed of several different sub-signals.
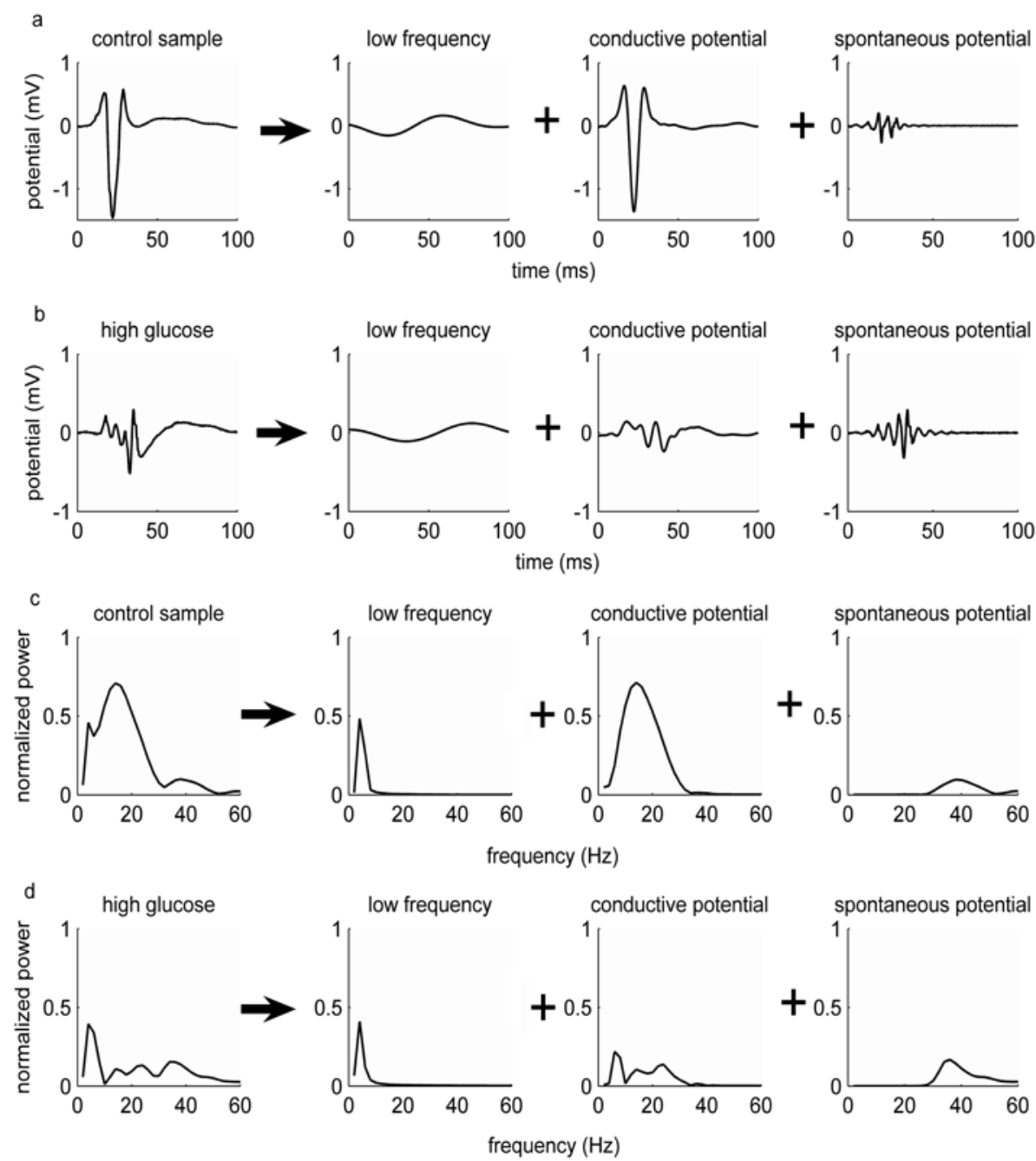

Fig. 2. Signal decomposition. (a) The field potentials of the control sample in the time domain. (b) The field potentials in high glucose environment in the time domain (The concentration of glucose was $40 \mathrm{mM}$, the actuation duration was 30 minutes). (c) The energy distributions of the control sample in the frequency domain. (d) The energy distributions in high glucose environment in the frequency domain (The concentration of glucose was $40 \mathrm{mM}$, the actuation duration was 30 minutes). 
Table 1

The predictive results of PLS and HOPLS in different concentrations of glucose and actuation durations

\begin{tabular}{|c|c|c|c|c|c|c|c|c|c|}
\hline \multirow[t]{2}{*}{ Concentration $(\mathrm{mM})$} & \multicolumn{2}{|l|}{ PLS } & \multicolumn{2}{|l|}{ HOPLS } & \multirow{2}{*}{$\begin{array}{l}\text { Actuation } \\
\text { duration (min) }\end{array}$} & \multicolumn{2}{|l|}{ PLS } & \multicolumn{2}{|l|}{ HOPLS } \\
\hline & RMSEP & $R_{P}^{2}$ & RMSEP & $R_{P}^{2}$ & & RMSEP & $R_{P}^{2}$ & RMSEP & $R_{P}^{2}$ \\
\hline 20 & 6.3911 & 0.4370 & \multirow[t]{5}{*}{6.3144} & \multirow[t]{5}{*}{0.8069} & 10 & 8.1791 & 0.4715 & \multirow[t]{5}{*}{6.2098} & \multirow[t]{5}{*}{0.8760} \\
\hline 30 & 5.3133 & 0.5989 & & & 15 & 6.2468 & 0.4529 & & \\
\hline 40 & 6.6031 & 0.3837 & & & 20 & 6.4046 & 0.5779 & & \\
\hline 50 & 5.4472 & 0.6895 & & & 25 & 5.5100 & 0.6501 & & \\
\hline & & & & & 30 & 6.3029 & 0.5093 & & \\
\hline
\end{tabular}

Note: RMSEP: root mean squared error of prediction; $R_{P}^{2}$ : squared correlation corefficient of prediction.

The sinoatrial node field potentials were separated into three sub-signals using wavelet analysis [15] (the wavelet function was Dmey and the decomposition level was 9). In this paper, the sub-signals were named as the low frequency field potential, conductive field potential and spontaneous field potential respectively. Figure 2 showed the signal decomposition in the time and frequency domains. In the high glucose environment, the three signals showed different sensitivities and did not disturb each other. They comprised the three-dimensional independent input tensor $\underline{\mathbf{X}}$. The concentrations and actuation durations of high glucose comprised the two-dimensional dependent output matrix $\mathbf{Y}$. Thus, a prediction model for the concentrations and actuation durations could be built by the method in Part 2.1 .

\subsection{Predictive abilities}

The predictive abilities of HOPLS and PLS were compared. PLS could not predict the concentration and actuation duration simultaneously since the output of PLS is a vector. Therefore, when PLS was used, the concentrations were fixed when the actuation durations were predicted and the actuation durations were fixed when the concentrations were predicted. Table 1 presented the predictive abilities of HOPLS and PLS for the concentrations and actuation durations of high glucose. The root-meansquared error of prediction (RMSEP) of concentration using HOPLS was 6.3144, which was $6.32 \%$ higher than the average value of the RMSEP using PLS, which was 5.9386. The RMSEP of actuation duration using HOPLS was 6.2098. This was 5.06\% lower than the average value of the RMSEP using PLS, which was 6.5409. The results indicated that under the condition of predicting the concentration and the actuation duration simultaneously, the predictive ability of HOPLS and PLS was in the same order of magnitude. Meanwhile, the squared correlation coefficient of prediction $\left(R_{p}^{2}\right)$ of HOPLS was higher than the one of PLS. It indicated that the dispersion degree of predicted values using HOPLS were lower.

In clinical and experimental studies, the common situation is that the concentration and actuation duration of high glucose are both unknown. In this case, PLS will be invalid since the output is a vector. HOPLS is suitable for this situation and its predictive ability is comparable to PLS's.

\section{Conclusion}

HOPLS provides a very effective method for building a prediction model with tensor-input and tensor-output. Sinoatrial node field potential is composed of three independent sub-signals. The signals are sensitive to high glucose. It is suitable to build a prediction model using HOPLS if the three sub-signals are regarded as the input tensor and the concentrations and actuation durations of 
high glucose are regarded as the output tensor. The predictive abilities of HOPLS and PLS were comparable, but HOPLS could predict multi-dimension variable.

\section{References}

[1] T. Meyer, K.H. Boven, E. Gunther and M. Fejtl, Micro-electrode arrays in cardiac safety pharmacology-A novel tool to study QT interval prolongation, Drug Safety 27 (2004), 763-772.

[2] C.J. Stam, G. Nolte and A. Daffertshofer, Phase lag index: Assessment of functional connectivity from multi channel EEG and MEG with diminished bias from common sources, Human Brain Mapping 28 (2007), 1178-1193.

[3] J. Shlens, G.D. Field, J.L. Gauthier, M.I. Grivich, D. Petrusca, A. Sher, A.M. Litke and E.J. Chichilnisky, The structure of multi-neuron firing patterns in primate retina, Journal of Neuroscience 26 (2006), 8254-8266.

[4] R. Ergon, PLS score-loading correspondence and a bi-orthogonal factorization, Journal of Chemometrics 16 (2002), 368373.

[5] R. Rosipal and L.J. Trejo, Kernel partial least squares regression in reproducing kernel Hilbert space, Journal of Machine Learning Research 2 (2002), 97-123.

[6] Z.Y. Zhou, W. Liu, J.L. Cui, X.H. Wang, D. Arias, Y. Wen, R. Bansal, X.J. Hao, Z.S. Wang, B.S. Peterson and D.R. Xu, Automated artifact detection and removal for improved tensor estimation in motion-corrupted DTI data sets using the combination of local binary patterns and 2D partial least squares, Magnetic Resonance Imaging 29 (2011), 230-242.

[7] Q. Zhao, C.F. Caiafa, D.P. Mandic, Z.C. Chao, Y. Nagasaka, N. Fujii, L. Zhang and A. Cichocki, Higher order partial least squares (HOPLS): A generalized multilinear regression method, IEEE Transactions on Pattern Analysis and Machine Intelligence 35 (2013), 1660-1673.

[8] Q. Huang and L. Li, Modeling individual HRTF tensor using high-order partial least squares, EURASIP Journal on Advances in Signal Processing 58 (2014).

[9] M.R. Boyett, H. Honjo and I. Kodama, The sinoatrial node, a heterogeneous pacemaker structure, Cardiovascular Research 47 (2000), 658-687.

[10]F.J. Sutherland and D.J. Hearse, The isolated blood and perfusion fluid perfused heart, Pharmacological Research 41 (2000), 613-627.

[11] K. Nishi, Y. Yoshikawa, F. Takenaka and N. Akaike, Electrical-activity of sinoatrial node cells of rabbit surviving a long exposure to cold tyrodes solution, Circulation Research 41 (1977), 242-247.

[12]E.E. Verheijck, M.J.A. van Kempen, M. Veereschild, J. Lurvink, H.J. Jongsma and L.N. Bouman, Electrophysiological features of the mouse sinoatrial node in relation to connexin distribution, Cardiovascular Research 52 (2001), 40-50.

[13] T. Naes, T. Isaksson and B. Kowalski, Locally weighted regression and scatter correction for near-infrared reflectance data, Analytical Chemistry 62 (1990), 664-673.

[14]P.V. Bayly, B.H. KenKnight, J.M. Rogers, R.E. Hillsley, R.E. Ideker and W.M. Smith, Estimation of conduction velocity vector fields from epicardial mapping data, IEEE Transactions on Biomedical Engineering 45 (1998), 563-571.

[15]H. Asfour, L.M. Swift, N. Sarvazyan, M. Doroslovacki and M.W. Kay, Signal decomposition of transmembrane voltagesensitive dye fluorescence using a multiresolution wavelet analysis, IEEE Transactions on Biomedical Engineering $\mathbf{5 8}$ (2011), 2083-2093. 